\title{
Head and Neck Squamous Cell Cancer: Approach to Staging and Surveillance
}

\author{
Christine M. Glastonbury
}

\section{Learning Objectives}

- To understand the development of HN SCC arising from different areas in the $\mathrm{HN}$ and particularly the contributions of viruses and environmental exposure factors in its pathogenesis.

- To recognize the important features of the AJCC/ UICC staging system and how radiologists can use this system to provide more detailed, valuable reports.

- To develop a systematic method for evaluating all post-treatment scans from baseline through surveillance in order to detect residual, recurrent, and new $\mathrm{HN}$ malignancies.

\subsection{Imaging Approaches for HN SCC}

Across the USA and across the world there are different thoughts and perspectives as to the best imaging modality to use for staging and surveillance, but also as to the ideal timing for use of the modalities after treatment and for ongoing surveillance. The AJCC offers some guidance about the utility of imaging modalities for the staging of specific entities but offers no surveillance plan [1]. In the USA the National Comprehensive Cancer Network [NCCN] guidelines for HN cancer treatment sets out broad strokes but these surveillance protocols are variably used and were developed without radiologist input [2]. The NI-RADS reporting system for

\section{M. Glastonbury $(\bowtie)$}

Department of Radiology and Biomedical Imaging,

University of California, San Francisco, CA, USA

Department of Otolaryngology-Head and Neck Surgery and Department of Radiation Oncology, University of California, San Francisco, CA, USA

e-mail: Christine.glastonbury@ucsf.edu baseline and surveillance imaging after treatment was modeled after the breast imaging BI-RADS system, and aims to standardize an imaging approach to HN cancer with management recommendations. Through ongoing studying of the results of this system, we seek to determine the accuracy of imaging and then further optimize surveillance algorithms in $\mathrm{HN}$ cancer [3-5].

When staging HN SCC some specific tumor sites are better served by either CT or MR. At different hospitals and imaging sites modalities may be favored because of cost, modality, availability, or patient tolerance for the imaging exam. Excellent quality whole neck imaging is more readily reproducible from patient to patient using CT than MR, and this is particularly true for the post-treatment patient who may have difficulty lying on a MR for an extended period while dealing with oral secretions. MR and PET are more expensive and may not be readily accessible in different locations in the USA because of insurance costs. Large field of view, non-optimized MR sequences and lack of familiarity with basic neck anatomy or MR artifacts will make detection of key findings difficult for the less experienced radiologist.

With these caveats in mind, MR offers specific utility in certain areas. For example, it is the preferred staging tool for nasopharyngeal carcinoma because the detection of skull base infiltration (T3) or intracranial extension of disease (T4) is extremely important for staging and treatment planning. Likewise, MR offers significantly better soft tissue contrast for detecting small primary tonsillar tumors and evaluating the deep extent of an infiltrative lesion when planning surgical resection or intensity modulated radiation therapy (IMRT). For this reason it is often used in the oral cavity and oropharynx. In the larynx, MR is so affected by motion artifact that it is largely reserved for determination of cartilage penetration (T4a) when CT is equivocal. Finally, nodal disease at any site is almost equally well evaluated with either CT or MR.

Given the complexity of neck anatomy and intimately located structures, FDG-PET in the HN is best performed as a combined PET/CT examination. It is important to be 
aware of the many tissues with variable degrees of normal FDG uptake, particularly muscles, brown fat, salivary and lymphoid tissue, and recent biopsy sites. These all serve as potential false-positive pitfalls in PET imaging. A potential false-negative finding is the absence of uptake in a predominantly cystic node. Correlation with neck CT imaging will allow correct identification of cystic nodal metastases.

Ultrasound (US) has a limited but important role in $\mathrm{HN}$ SCC. At many practices in the USA it is largely reserved for interrogation of nodes that are equivocal on other imaging modalities, and particularly when a positive finding, such as a contralateral node, might significantly alter staging and management. US can also serve as imaging guidance for fine needle aspiration (FNA).

\subsection{Imaging Anatomy for HN SCC}

It is from the squamous lining of the pharynx, larynx, and sinonasal cavity that mucosal SCC arises. The pharynx is really a muscular tube, extending from the skull base where it is attached by the pharyngobasilar fascia, to the larynx and cervical esophagus. Staging of SCC is individualized to each site, and, in the case of laryngeal SCC, to each of its three subsites $[1,6]$.

The nasopharynx is so-named as it is located posterior to the nasal cavity, extending from the most cranial aspect of the pharynx at the skull base to the level of the soft palate. Most nasopharyngeal carcinomas arise from the lateral pharyngeal recess, also known as the fossa of Rosenmüller, which is adjacent to the opening of the Eustachian tube, and thus nasopharyngeal tumors often result in middle ear effusion at the time of presentation. In the midline of the nasopharynx, immediately caudal to the clivus is the nasopharyngeal tonsillar tissue ["adenoids"] from which nasopharyngeal lymphoma may arise. Adenoidal hypertrophy most often occurs in response to viral pathogens including HIV infection, and can mimic nasopharyngeal neoplasms although there should be symmetry, distinction of this tissue from prevertebral muscles and parapharyngeal fat, and on T2-weighted MR imaging there should preservation of a mucosal stripe [7].

Inferiorly the nasopharynx is contiguous with the oropharynx, which extends caudally to the hyoid bone. The posterior wall of the nasopharynx becomes the posterior wall of the oropharynx. The anterior tonsillar pillars and the circumvallate papillae of the tongue define the anterior limit of the oropharynx. The posterior one third of the tongue is called the tongue base and is the site of the lingual tonsillar tissue. This is part of the oropharynx and tumors arising here are staged using one of two oropharyngeal tumor staging systems depending on their pathologic p16 or high-risk HPV status $[1,6]$. The anterior two thirds of the tongue is known as the oral tongue and lies in the oral cavity. Tumors arising from the inner mucosal surface of the lips, the gingiva of the maxilla and mandible, the hard palate, and the cheek lining [buccal mucosa] are all classified as oral cavity tumors and staged using the oral cavity staging forms.

Below the hyoid bone, the pharynx divides into the more anterior larynx airway, and the posterior hypopharynx for digestion. The posterior wall of the oropharynx continues as the posterior wall of the hypopharynx while the anterior wall of the hypopharynx is the post-cricoid region, the mucosa overlying the posterior surface of the cricoid cartilage of the larynx. The lateral recesses or "pockets" of the hypopharynx are the pyriform sinuses, from which nearly two thirds of hypopharyngeal SCC arise. The aryepiglottic (AE) folds separate the hypopharynx from the supraglottic larynx and tumors arising from these folds are staged as supraglottic tumors. The inferior aspect of the hypopharynx is at the inferior aspect of the cricopharyngeus muscle, which marks the beginning of the cervical esophagus. On cross-sectional imaging this can be determined by the inferior aspect of the cricoid cartilage, and on axial sections the semilunar shape of the collapsed hypopharynx abruptly transitions to the rounded contour of the muscular esophagus [8].

The larynx connects the oropharynx airway to the tracheal airway and is more anterior in the neck. Embryologically, and for tumor staging, it is divided into three subsites, each of which has a separate staging table. The glottis is the true vocal cords; muscular structures which give rise to voice and which extend from the arytenoid cartilages to the midline thyroid cartilage anteriorly. More than half of all laryngeal SCC arise from the mucosal surface of the glottis. The supraglottic larynx is comprised of the structures superior to this including the epiglottis, AE folds, and the false cords. The epiglottis has both laryngeal and lingual surfaces and its most superior aspect extends above the level of the hyoid bone although all tumors arising from the mucosal aspect of the epiglottis are considered supraglottic. The subglottis is the mucosal lining internal to the cricoid cartilage ring below the glottis. The subglottis is contiguous with the cervical trachea and tumors of this area are the least common of all laryngeal SCC.

When talking about HN SCC the nasal cavity and paranasal sinuses are not often included in the discussion; however, they comprise up to $5 \%$ of all $\mathrm{HN}$ cancers and up to $70 \%$ of these are mucosal SCC [9]. The nasal cavity is anterior to the nasopharynx and from this branch the paired left and right paranasal sinuses: frontal, maxillary, sphenoid, and the anterior and posterior ethmoid air cells. Sinonasal SCC are not associated with tobacco, but have been associated with industrial exposure such as wood, nickel dust, or formaldehyde [10, 11]. More recently it has been determined that approximately $25 \%$ of all sinonasal SCC are associated with high-risk HPV [9]. 


\subsection{Staging HN SCC}

In 2018 the eighth edition AJCC/UICC tumor classification system was released after several years of collaborative work between the two committees and from evaluation of interval research that better understood the molecular underpinnings and the prognosis of HN SCC $[1,6]$. While there have been many changes to staging in HN SCC it is not necessary to remember all the intricacies of staging tables. It is more important that the radiologist is aware that such tables exist and that when reporting imaging studies, reference to the specific criteria that determine the tumor $(\mathrm{T})$, nodal $(\mathrm{N})$, and metastasis (M) designation greatly enhances an imaging report by providing critical tumor information $[12,13]$. It is ideal to have on hand a copy of the staging tables to ensure that information is included in the report, keeping in mind that it is not the radiologist's role to provide a TNM stage, but rather to provide all information so that this can be done by the managing physician.

When evaluating the primary tumor $(\mathrm{T})$ the specific criteria in the tables may require distinction by size of the tumor or by specific invasion patterns. When measuring tumor size the longest diameter is used. It is important to refer to $\mathrm{T}$ criteria for specific areas of invasion in order to include such relevant positive or negative information in the radiology report. Some criteria will not be known to the radiologist such as impairment of vocal cord mobility in laryngeal tumors, and depth of invasion (DOI) for oral cavity tumors, which is purely a pathological measurement. Minor changes have been made to the $\mathrm{T}$ criteria for nasopharyngeal carcinoma and for oropharyngeal carcinoma and lip tumors have been removed from the oral cavity staging, and have their own $\mathrm{T}$ criterion under a new chapter of "cutaneous carcinoma of the head and neck."

The detection of perineural tumor (PNT) may significantly alter the extent of surgical resection and/or the radiation treatment field even if it does not change the $\mathrm{T}$ designation and so should be sought on all examinations. Both mucosal and skin SCC exhibit neurotropism as do other skin malignancies, salivary gland tumors, and lymphomas. PNT is usually more readily evident on MR but may be found on CT with careful evaluation of known routes of spread. Evidence of perineural tumor may significantly alter the primary surgical plan and/or the radiation field [14].

The most significant change to the AJCC/UICC staging is the bifurcation of oropharyngeal tumor staging into those tumors which are shown to be 16 positive by immunohistochemistry or shown to be high-risk HPV DNA positive, from those which are not. The former group of $\mathrm{p} 16+/$ HPV-associated oropharyngeal SCC have a markedly better prognosis despite their tendency to have early and extensive nodal metastasis $[15,16]$. The nodal table and the overall prognostic table for these tumors have been markedly modi- fied to reflect this, with up to $80 \%$ of these tumors now being classified as stage 1 with a $90 \% 5$-year survival. This is in contrast to p16-/non-HPV oropharyngeal SCC where most tumors are stage III or stage IV at presentation (Fig. 17.1).

The second major change to the eighth edition of the AJCC/UICC is alteration of the nodal staging table for all nonHPV and non-EBV related SCC and development of separate
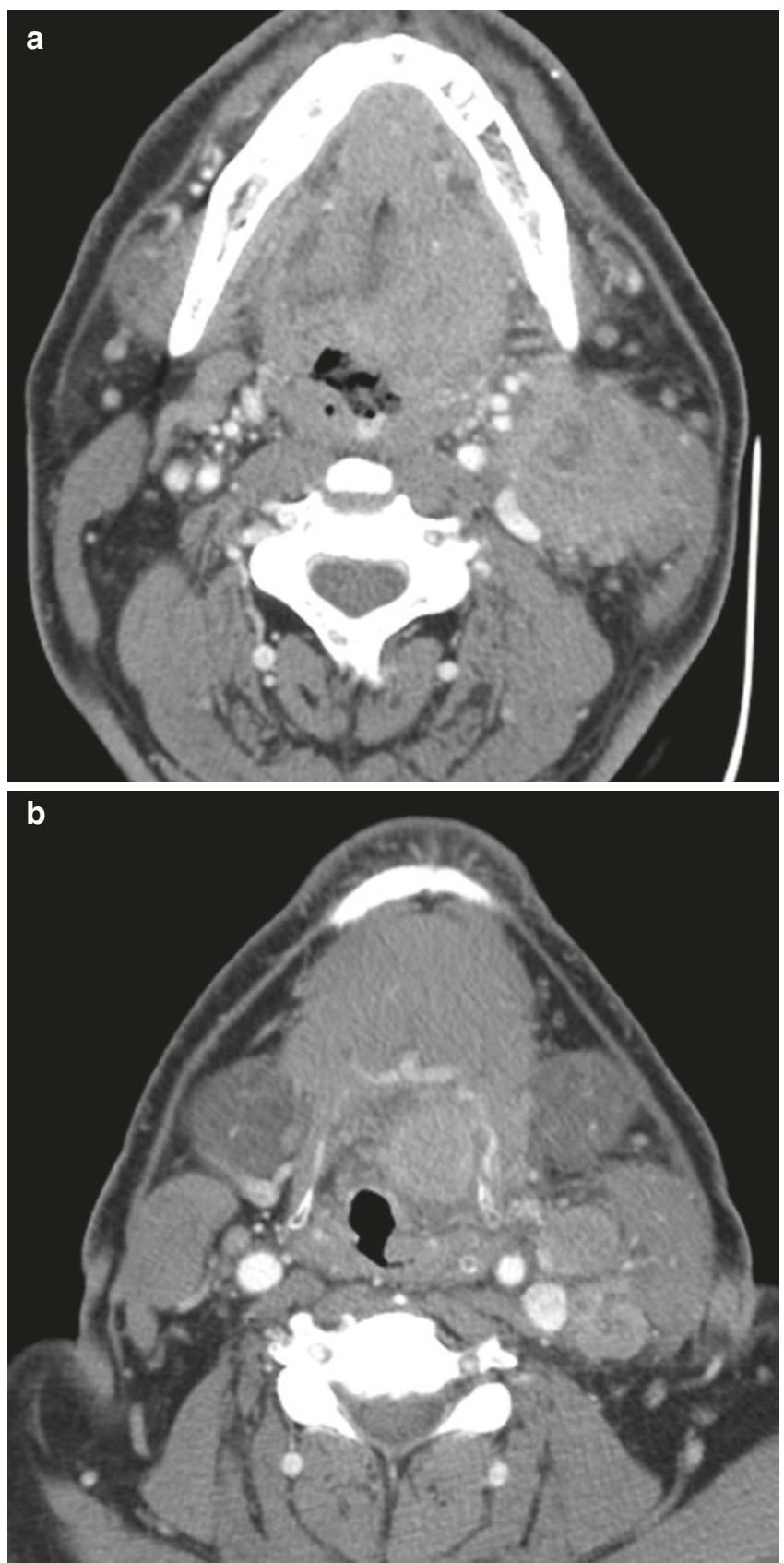

Fig. 17.1 A 51-year-old man with a 30-pack year smoking history presents with a left neck mass. The primary is a large infiltrating tumor at the left tongue base extending into the floor of mouth $(\mathbf{a}, \mathbf{b})$. There are multiple ipsilateral heterogeneous level 2 and 3 nodal masses with illdefined margins (b). As a p16+ SCC this was T4N1M0, stage III. If this had been p16- it would be classified as T4aN3bM0 and stage IVB 

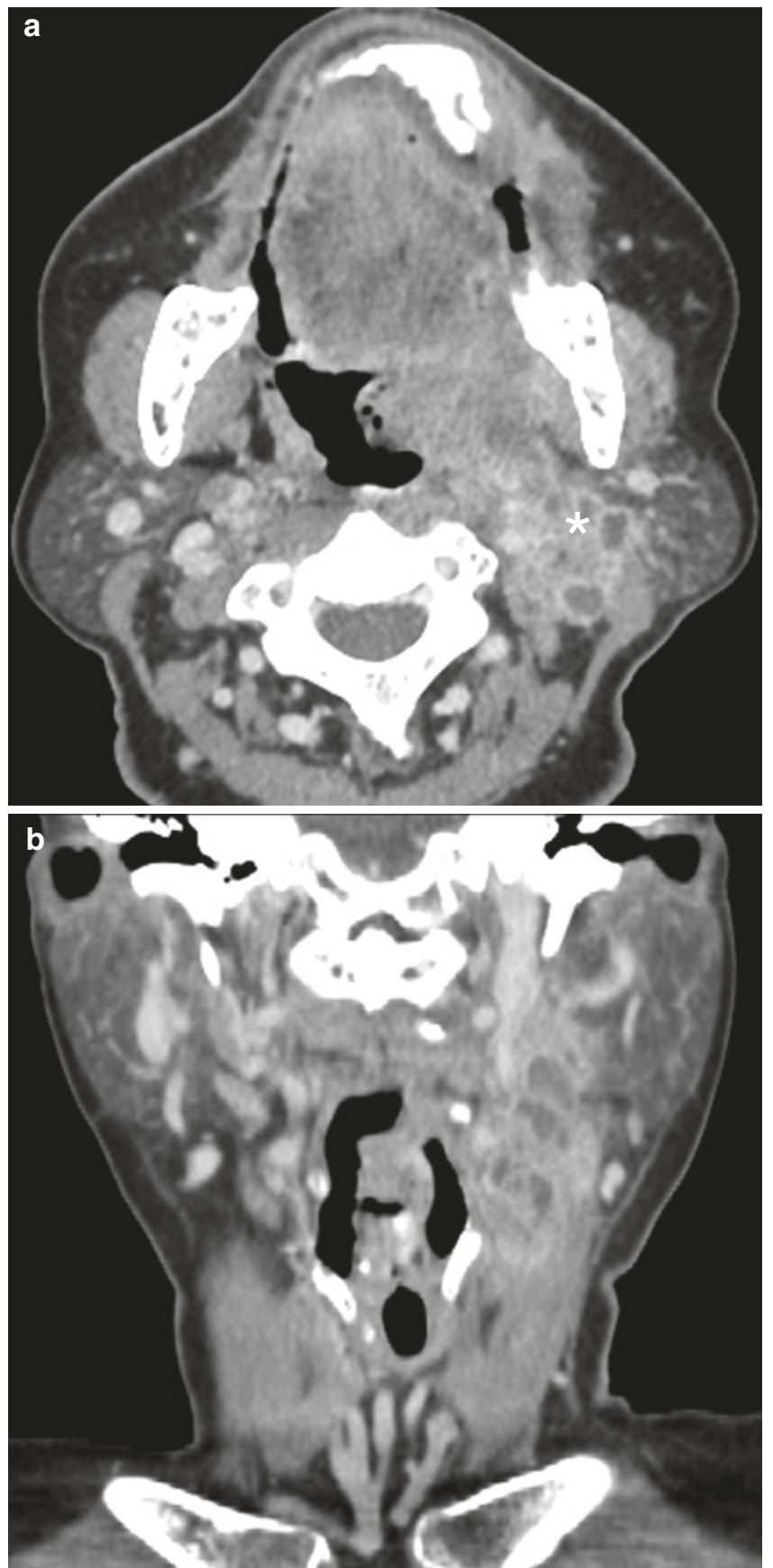

Fig. 17.2 Axial and coronal CECT in a 74-year-old woman presenting with a fixed left neck mass, representing clinical extranodal extension [cENE]. There is a large left tonsillar primary tumor and a nodal conglomerate at the left 2A nodal group (*). The nodal mass has ill-defined margins with infiltration of the adjacent sternocleidomastoid muscle and posterior triangle fat planes. Extranodal extension denotes N3b disease for all HN SCC except NPC and p16+/HPV-associated OPSCC where it is not part of the nodal criteria

pathologic tables for these tumors when neck dissections are performed. This has the potential for significant confusion for radiologists, particularly when the p16/HPV status is unknown for oropharyngeal tumors. In this situation, it is critical to care- fully evaluate the entire neck ipsilateral and contralateral to the primary site for any enlarged, heterogeneous, or frankly necrotic nodes and describe those that appear distinctly positive for tumor or which are highly suspicious. Extranodal extension of tumor (ENE) that is evident on clinical examination by the physician is now an important finding that upstages nodal disease to N3 for p16-/non-HPV and non-EBV lymph node SCC (Fig. 17.2). It has long been recognized that ENE is associated with a poorer prognosis and higher rate of tumor recurrence but it is only in this eighth edition that it has been included in the clinical and pathologic staging tables.

Evaluation for M1 disease (the presence of any metastasis) is best done with PET imaging although patients are always assumed to be M0 if no distant staging imaging/evaluation is performed. At the time of any staging neck scan, the lung apices and the bones should always be evaluated for metastases. Additionally, as many SCC are tobacco and alcohol-related, these patients carry an increased risk of a second primary neoplasm. Second primary tumors are most frequently found with hypopharyngeal SCC and 1/3 are synchronous with the initial SCC. Second primary tumors are most commonly another HN SCC or esophageal or pulmonary primary tumors.

\section{Key Points}

- When staging a new tumor start by locating the primary site and then reference the appropriate table in AJCC/UICC for critical points to include in the report.

- Tumors should always be measured by their longest diameter and then describe the deep extent and evaluate for perineural tumor.

- Evaluate the regional drainage nodes and always look for contralateral nodal metastases.

- Complete the neck report by evaluating for bone and lung metastases.

\subsection{Unknown Primary Tumors}

When a patient presents with a neck mass that is found to be nodal SCC, an initial clinical examination is performed in the ENT surgeon's office. If a primary site is not evident, this is considered to be an "unknown primary tumor" and imaging has an important role in trying to determine the origin so that biopsy can be directed. Radiation can also be targeted to the area of concern, thus reducing treatment morbidity. More than $90 \%$ of unknown primary SCC detected from FNA of a nodal mass in the absence of a clinically evident primary source, are due to $\mathrm{p} 16+/ \mathrm{HPV}$-associated oropharyngeal SCC [17]. It is thus critical to carefully evaluate the palatine and lingual tonsils which may harbor tumor in the depths of the tonsillar crypts where it may not be evident on clinical exam- 
ination. Thus subtle asymmetry of density/signal intensity or of size of the tonsil ipsilateral to a nodal mass suggests the most likely primary site and directs the surgeon to the preferred site for biopsy or tonsillectomy.

It is also important to be aware that nodal metastasis from a cutaneous SCC primary or from a pulmonary SCC can also be p16+ and this must also be considered in the differential when a nodal FNA reveals p16+ SCC. In situations such as these when there is no primary oropharyngeal tumor evident, the node should also be tested for high-risk HPV prior to assuming an occult oropharyngeal source. PET/CT has proven to be extremely useful for determination of the primary tumor site in the presence of an unknown primary nodal metastasis and is frequently the first-line imaging study when a patient presents without a clinical source evident.

If the nodal SCC is p16-, then other sites of origin should be considered including the nasopharynx and hence EBV testing should be performed. If this is still negative and no primary site is evident on cross-sectional CT, MR, or PET/ $\mathrm{CT}$, then no primary $\mathrm{T}$ designation is assigned.

In contrast to the situation where a new imaging study is ordered after FNA has proven that SCC is present, when a scan is ordered for a neck mass, any cystic or solid new mass in an adult should be assumed to be neoplastic until proven otherwise.

\subsection{Baseline and Surveillance Imaging of HN SCC}

Following surgery, radiation, and/or chemotherapy, a posttreatment baseline imaging study should be obtained and should be evaluated with knowledge of the primary site and
AJCC/UICC tumor stage, and the entire treatment plan. Baseline scans, particularly following reconstruction flaps or radiation can be extremely difficult to evaluate, but understanding the expected findings following such treatment is paramount to anticipate the sites and appearances of treatment change and therefore distinguish residual tumor and early recurrence. The baseline scan should show no evidence of residual primary mass or abnormal lymph nodes (Fig. 17.3). It is particularly important to carefully evaluate both the primary site and neck nodal levels when there has been an unexpectedly prolonged delay from treatment to baseline scanning due to complications from surgery or other treatment, or loss of the patient to follow-up.

There is no consensus on the timing of the baseline scan but in the USA it is typically obtained at 8-10 weeks following chemoradiation, while postsurgical scans are often obtained around 10-12 weeks. PET/CT should be delayed to around 12 weeks to minimize false-positive FDG uptake from post-treatment inflammatory changes. The baseline cross-sectional scan also serves as a "road map" of a deformed neck to increase sensitivity for early recurrent disease on the subsequent surveillance imaging. For this reason, it is ideal that the same cross-sectional modality chosen for baseline imaging, either CT or MR, is subsequently used for surveillance.

Radiation therapy has changed enormously over the last two decades with the increasing use of intensity modulated radiotherapy (IMRT) for head and neck cancers. IMRT maximizes dose to the tumor and minimizes radiation to unaffected tissues but requires accurate delineation of tumor margins. This is often best done with MR although some practices use PET/CT or CECT alone, and may also
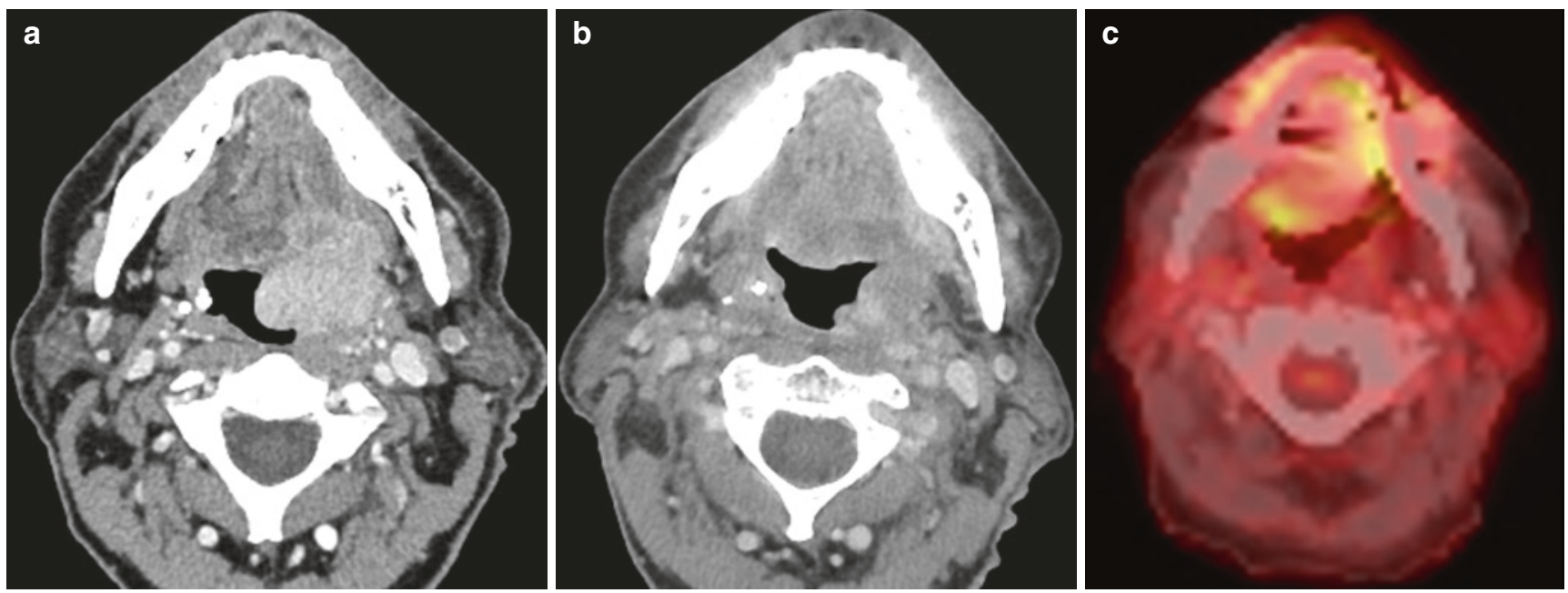

Fig. 17.3 A 66-year-old man with a large left palatine tonsillar mass staged as p16+ SCC cT4N0M0 stage III (a). The patient completed chemoradiation with baseline CT 8 weeks later (b) showing marked decrease in enhancement and decrease in bulk. This is not completely resolved as is hoped for on the baseline scan. Short interval follow-up PET/CT was obtained 4 weeks later (c) showing no significant uptake. The patient has remained disease free 
require greater input from radiologists to ensure accurate treatment volumes [18]. Radiation with or without chemotherapy results in significant changes in the appearance of neck soft tissues [19]. Radiation results in acute inflammation of all tissues in the radiation field with extensive edema. Over time this converts to fibrosis with atrophy and altered signal intensity and texture on MR. Both acute and chronic expected radiation changes can be confusing on $\mathrm{CT}$ or MR, requiring careful evaluation and comparison with prior studies to detect early recurrences.

Surgical resection of a primary tumor and/or nodal drainage regions also results in variable changes to normal neck contours. Familiarity with the types of nodal neck dissections and common flap reconstructions is necessary to evaluate for both complications and recurrence [20, 21]. It is extremely helpful to have knowledge of what surgical procedure was performed prior to evaluating such scans. Relatively simple resections, such as selective neck dissections, can be surprisingly subtle on imaging. Conversely, large resections with flap reconstructions can be quite complex, and imaging is hampered by metallic hardware. MR is less affected by hardware artifact and more sensitive for recurrent tumor, however, the muscular component of a flap reconstruction will undergo denervation change resulting in variable MR signal intensity and enhancement $[22,23]$. It is important on the baseline scan following neck reconstruction to ensure there is no residual or progressive tumor, and having knowledge of the pathology report and of positive tumor margins at the time of reconstruction can be very helpful in this regard.

Recurrent SCC most often occurs during the first 2 years following treatment. The frequency of surveillance imag- ing during this time is variable and may be performed in 3-6 month intervals, depending on the initial tumor staging and histologic prognostic features and the ongoing clinical course and physical findings. At any follow-up imaging examination, the possibility of a second primary tumor must again be considered. Remember on every follow-up study to look for residual, recurrent, and new tumors.

With NI-RADS, the timing for imaging after surgery or chemotherapy for HN cancer is a baseline CT or MRI at $8-12$ weeks with concurrent PET. If this is negative, neck imaging and chest $\mathrm{CT}$ is performed at 6 months, then further neck imaging at 6 months, and if this remains negative then neck and chest imaging at 8-12 months [24]. Any concerning but not definitive findings at any point in the series of follow-up imaging steps is classified as NIR-RADS 2 and leads to either shorter interval imaging (3 months), PET, or direct inspection. Such examples of NI-RADS 2 findings are focal superficial mucosal enhancement or focal mucosal FDG uptake, or discordance between the CT and PET (Fig. 17.4). The neck nodes also receive a NI-RADS score, with NI-RADS 2 most often leading to recommendation for PET imaging. Biopsy of the primary site or lymph nodes is recommended if there is strong concern for residual/ recurrent disease [NI-RADS 3] such as a new or enlarging, discrete nodule with robust enhancement or intense focal FDG uptake on PET. NI-RADS 4 is evidence of an already pathologically proven recurrence or definitive radiologic or clinical progression/recurrence that does not require biopsy. Using NI-RADS in your clinical practice not only allows ready communication with clinicians as to the degree of concern of an imaging finding for malignancy, but also generates data-mineable report. These will allow future optimization
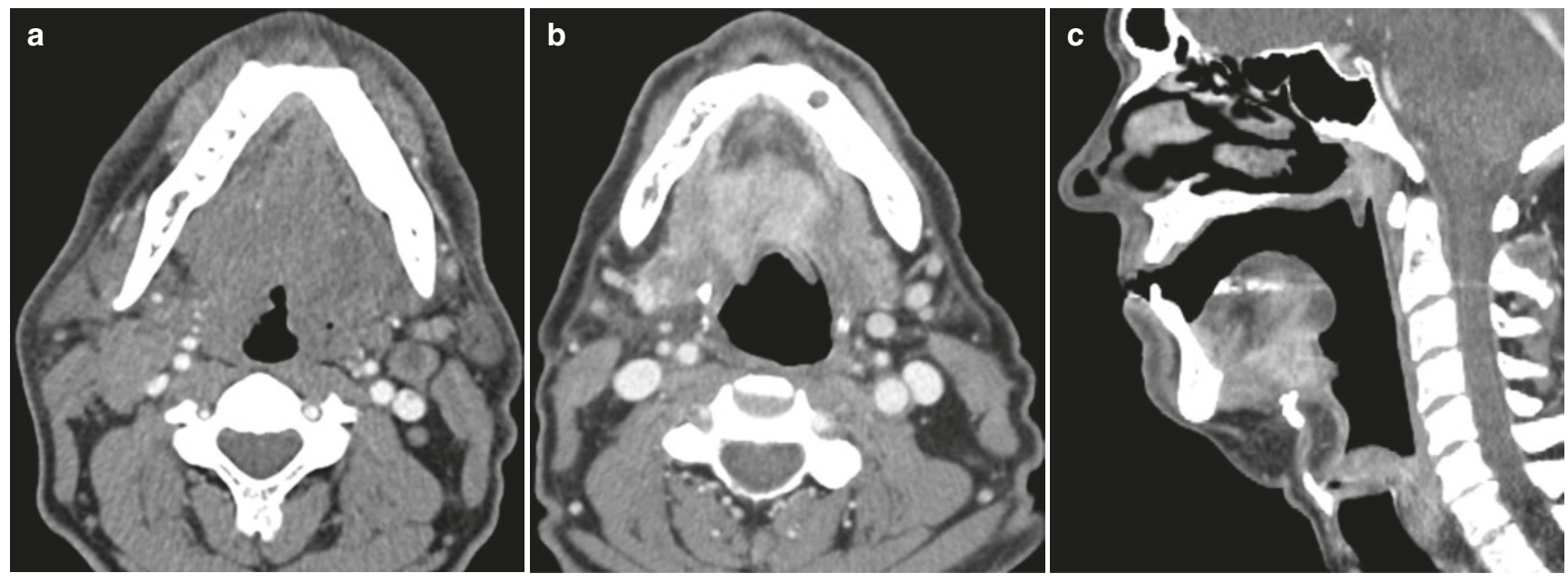

Fig. 17.4 A 59-year-old man diagnosed with p16+ base of tongue SCC T4N2M0 (a) after presenting with bilateral adenopathy and a poorly enhancing but infiltrating mass. The patient was treated with chemoradiation although did not receive all cycles of chemotherapy. Baseline imaging after completion of treatment $(\mathbf{b}, \mathbf{c})$ shows residual enhancing soft tissue mass in the floor of mouth but resolution of the adenopathy. This mass in the floor of the mouth is highly concerning for residual disease and should be biopsied. It is therefore a NI-RADS 3 . The resolved neck nodes are NI-RADS 1 
of imaging surveillance algorithms but also enable the user to determine their imaging accuracy and for practices to determine interobserver variability of reports.

\subsection{Concluding Remarks}

The radiologist has a valuable role in the management of $\mathrm{HN}$ SCC patients from initial staging or evaluation of a new neck mass, to post-treatment baseline imaging and surveillance. Post-treatment scans are often the most-complex examinations to interpret and so having a systematic process of performing the scans and reviewing the scans is important. It is essential for all post-treatment scans to have a history of the initial tumor type, the treatment performed, and the time interval of such. Further clarity can also be added to a report when there is additional knowledge of prior pathology reports, including surgical margins, and any clinical concerns for recurrent disease. The availability of much of this information via electronic medical records can greatly enhance the radiologist's sensitivity for subtle findings and confidence with approaching such cases.

\section{Take Home Messages}

- When reporting staging scans use the AJCC/UICC tables in order that the report includes critical criteria to enable the clinician to correctly stage the patient.

- HN post-treatment scans can be very difficult to read, but starting with a good history of the original tumor and the treatment plan set the stage for the best evaluation.

- Working with your clinicians to set a routine baseline and surveillance imaging plan and consistent imaging protocols is a good practice to standardize patient care and make radiologists most familiar with the expected appearance of treatment changes over time.

- Incorporating NI-RADS to the report can help your team gauge their accuracy and refine their reports.

- A new cystic or solid neck mass in an adult patient is cancer until proven otherwise.

\section{References}

1. Amin MB, Edge SB, Greene FL, et al., editors. AJCC cancer staging manual. 8th ed. New York: Springer; 2017.

2. National comprehensive cancer network guidelines. https://www. nccn.org/professionals/physician_gls/default.aspx\#site. Accessed 22 Sept 2019.

3. Aiken AH, Farley A, Baugnon KL, et al. Implementation of a novel surveillance template for head and neck cancer: neck imaging reporting and data system (NI-RADS). JACR. 2016;13:743-6.
4. Aiken AH, Rath TJ, Anzai Y, Branstetter BF, Hoang JK, Wiggins RH, Juliano AF, Glastonbury C, Phillips CD, Brown R, Hudgins PA. ACR neck imaging reporting and data systems (NI-RADS): a white paper of the ACR NI-RADS committee. J Am Coll Radiol. 2018;15(8):1097-108.

5. Krieger DA, Hudgins PA, Nayak GK, Baugnon KL, Corey AS, Patel MR, Beitler JJ, Saba NF, Liu Y, Aiken AH. Initial performance of NI-RADS to predict residual or recurrent head and neck squamous cell carcinoma. AJNR Am J Neuroradiol. 2017;38(6):1193-9.

6. Brierley JD, Gospodarowicz MK, Wittekind C, editors. TNM classification of malignant tumours. 8th ed. Hoboken: Wiley Blackwell; 2017.

7. Bhatia KS, King AD, Vlantis AC, Ahuja AT, Tse GM. Nasopharyngeal mucosa and adenoids: appearance at MR imaging. Radiology. 2012;263(2):437-43.

8. Schmalfuss IM, Mancuso AA, Tart RP. Postcricoid region and cervical esophagus: normal appearance at $\mathrm{CT}$ and MR imaging. Radiology. 2000;214(1):237-46.

9. Lewis JS Jr. Sinonasal squamous cell carcinoma: a review with emphasis on emerging histologic subtypes and the role of human papillomavirus. Head Neck Pathol. 2016;10(1):60-7.

10. Luce D, Leclerc A, Bégin D, Demers PA, Gérin M, Orlowski E, Kogevinas M, Belli S, Bugel I, Bolm-Audorff U, Brinton LA, Comba P, Hardell L, Hayes RB, Magnani C, Merler E, PrestonMartin S, Vaughan TL, Zheng W, Boffetta P. Sinonasal cancer and occupational exposures: a pooled analysis of 12 case-control studies. Cancer Causes Control. 2002;13(2):147-57.

11. Luce D, Gérin M, Leclerc A, Morcet JF, Brugère J, Goldberg M. Sinonasal cancer and occupational exposure to formaldehyde and other substances. Int J Cancer. 1993;53(2):224-31.

12. Glastonbury CM, Bhosale PR, Choyke PL, D’Orsi CJ, Erasmus JJ, Gill RR, Mukherji SK, Panicek DM, Schwartz LH, Subramaniam RM, Sullivan DC. Do radiologists have stage fright? Tumor staging and how we can add value to the care of patients with cancer. Radiology. 2016;278(1):11-2.

13. Glastonbury CM, Mukherji SK, O’Sullivan B, Lydiatt WM. Setting the stage for 2018: how the changes in the American joint committee on cancer/union for international cancer control cancer staging manual eighth edition impact radiologists. AJNR Am J Neuroradiol. 2017;38(12):2231-7.

14. Bakst RL, Glastonbury CM, Parvathaneni U, Katabi N, Hu KS, Yom SS. Perineural invasion and perineural tumor spread in head and neck cancer. Int J Radiat Oncol Biol Phys. 2019;103(5):1109-24.

15. O'Sullivan B, Huang SH, Su J, Garden AS, Sturgis EM, et al. Development and validation of a staging system for HPV-related oropharyngeal cancer by the international collaboration on oropharyngeal cancer network for staging (ICON-S): a multicentre cohort study. Lancet Oncol. 2016;17(4):440-51.

16. Haughey BH, Sinha P, Kallogjeri D, et al. Pathology-based staging for HPV positive squamous carcinoma of the oropharynx. Oral Oncol. 2016;62:11-9.

17. Motz K, Qualliotine JR, Rettig E, Richmon JD, Eisele DW, Fakhry C. Changes in unknown primary squamous cell carcinoma of the head and neck at initial presentation in the era of human papillomavirus. JAMA Otolaryngol Head Neck Surg. 2016;142(3):223-8.

18. Braunstein S, Glastonbury CM, Chen J, Quivey JM, Yom SS. Impact of neuroradiology-based peer review on head and neck radiotherapy target delineation. AJNR Am J Neuroradiol. 2017;38(1):146-53.

19. Glastonbury CM, Parker EE, Hoang JK. The postradiation neck: evaluating response to treatment and recognizing complications. AJR Am J Roentgenol. 2010;195(2):W164-71.

20. Hudgins PA, Kingdom TT, Weissler MC, Mukherji SK. Selective neck dissection: CT and MR imaging findings. AJNR Am J Neuroradiol. 2005;26(5):1174-7. 
21. Lobert P, Srinivasan A, Shah GV, Mukherji SK. Postoperative and postradiation changes on imaging. Otolaryngol Clin N Am. 2012;45(6):1405-22.

22. Syed F, Spector ME, Cornelius R, Srinivasan A. Head and neck reconstructive surgery: what the radiologist needs to know. Eur Radiol. 2016;26(10):3345-52.

23. McCarty JL, Corey AS, El-Deiry MW, Baddour HM, Cavazuti BM, Hudgins PA. Imaging of surgical free flaps in head and neck reconstruction. AJNR Am J Neuroradiol. 2019;40(1):5-13.

24. Aiken AH, Hudgins PA. Neck imaging reporting and data system. Magn Reson Imaging Clin N Am. 2018;26(1):51-62.

\section{Suggested Reading}

Chegini S, Mitsimponas K, Shakib K. A review of recent advances in histopathological assessment of head and neck squamous cell carcinoma. J Oral Pathol Med. 2019;00:1-5. https://doi.org/10.1111/ jop. 12943 .
Gurudutt VV, Genden EM. Cutaneous squamous cell carcinoma of the head and neck. J Skin Cancer. 2011;2011:502723.

Ouyang YH. Skin cancer of the head and neck. Semin Plast Surg. 2010;24(2):117-26.

Gandini S, Botteri E, Iodice S, Boniol M, Lowenfels AB, Maisonneuve P, Boyle P. Tobacco smoking and cancer: a meta-analysis. Int J Cancer. 2008;122(1):155-64.

Hashibe M, Boffetta P, Zaridze D, et al. Evidence for an important role of alcohol- and aldehyde-metabolizing genes in cancers of the upper aerodigestive tract. Cancer Epidemiol Biomark Prev. 2006;15(4):696-703.

Hashibe M, Brennan P, Benhamou S, et al. Alcohol drinking in never users of tobacco, cigarette smoking in never drinkers, and the risk of head and neck cancer: pooled analysis in the international head and neck Cancer epidemiology consortium. J Natl Cancer Inst. 2007;99(10):777-89.

National cancer institute facts and figures. https://seer.cancer.gov/ statfacts/html/oralcav.html. Accessed 22 Sept 2019.

Open Access This chapter is licensed under the terms of the Creative Commons Attribution 4.0 International License (http://creativecommons. org/licenses/by/4.0/), which permits use, sharing, adaptation, distribution and reproduction in any medium or format, as long as you give appropriate credit to the original author(s) and the source, provide a link to the Creative Commons license and indicate if changes were made.

The images or other third party material in this chapter are included in the chapter's Creative Commons license, unless indicated otherwise in a credit line to the material. If material is not included in the chapter's Creative Commons license and your intended use is not permitted by statutory regulation or exceeds the permitted use, you will need to obtain permission directly from the copyright holder. 